\title{
Mixed Type Higher Order Symmetric Duality Over Cones
}

\author{
Khushboo Verma, Pankaj Mathur, and T. R. Gulati
}

\begin{abstract}
In this paper, a new mixed type higher-order symmetric duality in scalar programming over cone is formulated. The weak, strong and converse duality theorems are proved for these programs under $\eta$-invexity $/ \eta$-pseudoinvexity assumptions. Self duality also discussed. As a special case of our duality relation, we give some known duality results. Our results generalize these existing dual formulations.
\end{abstract}

Index Terms-Higher-order symmetric duality, duality theorems, higher-order invexity/generalized invexity.

\section{INTRODUCTION}

Mangasarian [1] introduced the concept of second and higher-order duality for nonlinear problems. The study of higherorder duality is significant due to the computational advantage over the first order duality as it provides tighter bounds for the value of the objective function when approximations are used. Mond and Zhang [2] obtained duality results for various higher-order dual problems under higher-order invexity assumptions, Chen [3] presented Mond-Weir type higher order symmetric duality for scalar and multiobjective nondifferentiable programming problem under F-convexity while Mishra and Rueda [4] generalized Mangasarian [5] and Mond-Weir [6] type higher-order duality to higher-order type I functions. Ahmad et al. [6] discussed higher-order duality in nondifferentiable Multiobjective Programming. Recently, Ahmad [7] Unified higher-order duality in nondifferentiable multiobjective programming involving cones.

$\mathrm{Xu}$ [8] formulated two mixed type duals in multiobjective programming and also proved duality theorems. Ahmad and Husain [9] studied mixed symmetric multiobjective dual programs and obtained duality results under K-preinvexity and K-pseudoinvexity assumptions. Chandra et al. [10] and Yang et al. [11] discussed a mixed symmetric dual formulation for a nonlinear programming problem and for a class of nondifferentiable nonlinear programming problems, respectively. Later on, Ahmad [12] formulated mixed type symmetric dual in multiobjective programming problems ignoring nonnegativity restrictions of Bector et al. [13].

In this paper, a new mixed type higher-order symmetric duality over cone in multiobjective programming is formulated. The weak, strong and converse duality theorems

Manuscript received January 9, 2017; revised April 14, 2017. The research of the author Khushboo Verma is supported by the Department of Atomic Energy, Govt. of India, under the NBHM Post-Doctoral Fellowship Program No. 2/40 (31)/ 2015/ RD-II/9474.

Khushboo Verma and Pankaj Mathur are with the Department of Mathematics and Astronomy, University of Lucknow, Lucknow-226001, India (e-mail: 1986khushi@gmail.com,pankaj_mathur14@yahoo.co.in).

T. R. Gulati is with the Department of Mathematics, Indian Institute of Technology Roorkee, Roorkee-247 667, India (e-mail: trgulati@gmail.com). are proved for these programs under $\eta$-invexity/ $\eta$-pseudoinvexity assumptions. Self duality also discussed. As a special case of our duality relation, we give some known duality results. Special cases are discussed to show that this study extends some of the known results in [14], [15] and [4]

\section{PREREQUISITES}

For $N=\{1,2,3, \ldots, n\}$ and $M=\{1,2,3, \ldots, m\}$, let $J_{1} \subset N, K_{1} \subset M$ and $J_{2}=N \backslash J_{1}$ and $K_{2}=M \backslash K_{1}$. Let $\left|J_{1}\right|$ denote the number of elements in the set $J_{1}$. The other numbers $\left|J_{2}\right|,\left|K_{1}\right|$ and $\left|K_{2}\right|$ are defined similarly. Notice that if $J_{1}=\varnothing$, then $J_{2}=N$, that is $\left|J_{1}\right|=0$ and $\left|J_{2}\right|=n$. Hence, $R^{\left|J_{1}\right|}$ is zero dimensional Euclidean space and $R^{\left|J_{2}\right|}$ is n-dimensional Euclidean space. It is clear that any $\quad x \in R^{n} \quad$ can be written as $x=\left\{x^{1}, x^{2}\right\}, x^{1} \in R^{\left|J_{1}\right|}, x^{2} \in R^{\left|J_{2}\right|} \quad$ Similarly, any $y \in R^{m} \quad$ can be written as $y=\left\{y^{1}, y^{2}\right\}, y^{1} \in R^{\left|K_{1}\right|}, y^{2} \in R^{\left|K_{2}\right|}$

We consider the following programming problem :

(P) Minimize $F(x)$,

$$
-g(x) \in Q, \quad x \in S
$$

where $S \subseteq R^{n+m}$ and $F: S \rightarrow R$ and $\mathrm{Q}$ is a closed convex cone.

The following convention for vector inequalities will be used: If $\mathrm{a}, \mathrm{b} \in R^{n}$, then

$$
\begin{aligned}
& a \geq b \Leftrightarrow a_{i} \geq b_{i}, i=1,2, \ldots, n ; \\
& a \geq b \Leftrightarrow a \geq b \text { and } a \neq b ; \\
& a>b \Leftrightarrow a_{i}>b_{i}, i=1,2, \ldots, n .
\end{aligned}
$$

Definition 2.1 A function $\phi: S \mapsto R$ is said to be higher-order invex at $u \in S$ with respect to $\eta: S \times S \mapsto S$ and $h: S \times S \mapsto R, \quad$ if $\quad$ for $\operatorname{all}(x, p) \in S \times S, \begin{aligned} & \phi(x)-\phi(u)-h(u, p)+p^{T} \nabla_{p} h(u, p) \geqq \\ & \eta^{T}(x, u)\left\{\nabla_{x} \phi(u)+\nabla_{p} h(u, p)\right\} .\end{aligned}$

Definition 2.2 A function $\phi: S \mapsto R$ is said to be higher-order pseudoinvex at $u \in R^{n}$ with respect to $\eta: S \times S \mapsto S \quad$ and $\quad h: S \times S \mapsto R, \quad$ if $\quad$ for all 


$$
\begin{aligned}
(x, p) \in S \times S, & \eta^{T}(x, u)\left\{\nabla_{x} \phi(u)+\nabla_{p} h(u, p)\right\} \geqq 0 \\
& \Rightarrow \phi(x)-\phi(u)-h(u, p)+p^{T} \nabla_{p} h(u, p) \geqq 0 .
\end{aligned}
$$

Unless otherwise stated, $C_{1}, C_{2}, C_{3}$ and $C_{4}$ represent closed convex cones in $R^{\left|J_{1}\right|}, R^{\left|J_{2}\right|}, R^{\left|K_{1}\right|}$ and $R^{\left|K_{2}\right|}$, respectively, with non-empty interiors and $C_{i}^{*}, i=1,2,3,4$ is its polar cones and $S_{1} \subset R^{n}$ and $S_{2} \subset R^{m}$ are open sets such that $C_{1} \times C_{2} \subset S_{1} \times S_{2}$.

\section{HIGHER-ORDER MIXED TYPE SYMMETRIC DUALITY}

We consider the following pair of higher order symmetric duals and establish weak, strong and converse duality theorems.

Primal Problem (MHPC):

Minimize $L(x, y, p)=$ $f_{1}\left(x^{1}, y^{1}\right)+f_{2}\left(x^{2}, y^{2}\right)+h_{1}\left(x^{1}, y^{1}, p^{1}\right)+h_{2}\left(x^{2}, y^{2}, p^{2}\right)$ $-\left(p^{1}\right)^{T} \nabla_{p^{1}} h_{1}\left(x^{1}, y^{1}, p^{1}\right)-\left(p^{2}\right)^{T} \nabla_{p^{2}} h_{2}\left(x^{2}, y^{2}, p^{2}\right)$ $-\left(y^{2}\right)^{T}\left[\nabla_{y^{2}} f\left(x^{2}, y^{2}\right)+\nabla_{p^{2}} h_{2}\left(x^{2}, y^{2}, p^{2}\right)\right]$

Subject to

$$
\begin{gathered}
\nabla_{y^{1}} f\left(x^{1}, y^{1}\right)+\nabla_{p^{1}} h_{1}\left(x^{1}, y^{1}, p^{1}\right) \in C_{3}^{*}, \\
\nabla_{y^{2}} f\left(x^{2}, y^{2}\right)+\nabla_{p^{2}} h_{2}\left(x^{2}, y^{2}, p^{2}\right) \in C_{4}^{*}, \\
\left(y^{1}\right)^{T}\left[\nabla_{y^{1}} f\left(x^{1}, y^{1}\right)+\nabla_{p^{1}} h_{1}\left(x^{1}, y^{1}, p^{1}\right)\right] \geqq 0, \\
\left(p^{1}\right)^{T}\left[\nabla_{y^{1}} f\left(x^{1}, y^{1}\right)+\nabla_{p_{1}} h_{1}\left(x^{1}, y^{1}, p^{1}\right)\right] \geqq 0, \\
\left(p^{2}\right)^{T}\left[\nabla_{y^{2}} f\left(x^{2}, y^{2}\right)+\nabla_{p^{2}} h_{2}\left(x^{2}, y^{2}, p^{2}\right)\right] \geqq 0 \\
x^{1} \in C_{2}, x^{2} \in C_{2}, y^{2} \geqq 0,
\end{gathered}
$$

\section{Dual Problem (MHDC):}

Minimize $L(x, y, p)=$

$$
\begin{aligned}
& f_{1}\left(u^{1}, v^{1}\right)+f_{2}\left(u^{2}, v^{2}\right)+g_{1}\left(u^{1}, v^{1}, r^{1}\right)+g_{2}\left(u^{2}, v^{2}, r^{2}\right) \\
& -\left(r^{1}\right)^{T} \nabla_{r^{1}} g_{1}\left(u^{1}, v^{1}, r^{1}\right)-\left(r^{2}\right)^{T} \nabla_{r^{2}} g_{2}\left(u^{2}, v^{2}, r^{2}\right) \\
& -\left(u^{2}\right)^{T}\left[\nabla_{u^{2}} f^{2}\left(u^{2}, v^{2}\right)+\nabla_{r^{2}} g_{2}\left(u^{2}, v^{2}, r^{2}\right)\right]
\end{aligned}
$$

Subject to

$$
\begin{gathered}
\nabla_{u^{1}} f_{1}\left(u^{1}, v^{1}\right)+\nabla_{r^{1}} g_{1}\left(u^{1}, v^{1}, r^{1}\right) \in C_{1}^{*}, \\
\nabla_{u^{2}} f^{2}\left(u^{2}, v^{2}\right)+\nabla_{r^{2}} g_{2}\left(u^{2}, v^{2}, r^{2}\right) \in C_{2}^{*}, \\
\left(u^{1}\right)^{T}\left[\nabla_{u^{1}} f_{1}\left(u^{1}, v^{1}\right)+\nabla_{r^{1}} g_{1}\left(u^{1}, v^{1}, r^{1}\right)\right] \leqq 0, \\
\left(r^{1}\right)^{T}\left[\nabla_{u^{1}} f_{1}\left(u^{1}, v^{1}\right)+\nabla_{r^{1}} g_{1}\left(u^{1}, v^{1}, r^{1}\right)\right] \leqq 0, \\
\left(r^{2}\right)^{T}\left[\nabla_{u^{2}} f^{2}\left(u^{2}, v^{2}\right)+\nabla_{r^{2}} g_{2}\left(u^{2}, v^{2}, r^{2}\right)\right] \leqq 0, \\
v^{1} \in C_{3}, v^{2} \in C_{4}, u^{2} \geqq 0,
\end{gathered}
$$

where
(i) $f^{1}: R^{\left|J_{1}\right|} \times R^{\left|K_{1}\right|} \rightarrow R$,
(ii) $f^{2}: R^{\left|J_{2}\right|} \times R^{\left|K_{2}\right|} \rightarrow R$,
(iii) $g^{1}: R^{\left|J_{1}\right|} \times R^{\left|K_{1}\right|} \rightarrow R$,
(iv) $g^{2}: R^{\left|J_{2}\right|} \times R^{\left|K_{2}\right|} \rightarrow R$,
(v) $h^{1}: R^{\left|J_{1}\right|} \times R^{\left|K_{1}\right|} \times R^{\left|K_{1}\right|} \rightarrow R$,

(vi) $\quad h^{2}: R^{\left|J_{2}\right|} \times R^{\left|K_{2}\right|} \times R^{\left|K_{2}\right|} \rightarrow R \quad, \quad$ are twice differentiable functions, respectively,

(vii) $p^{1} \in R^{\left|K_{1}\right|}, \quad p^{2} \in R^{\left|K_{2}\right|}, \quad r^{1} \in R^{\left|J_{1}\right|}$ and $r^{2} \in R^{\left|J_{2}\right|}$.

\section{DUALITY THEOREMS}

\section{Theorem 4.1 (Weak Duality).}

Let $\left(x^{1}, x^{2}, y^{1}, y^{2}, p^{1}, p^{2}\right)$ be feasible for (PP) and $\left(u^{1}, u^{2}, v^{1}, v^{2}, r^{1}, r^{2}\right)$ be feasible for (DP). Suppose that

(i) $f^{1}\left(., v^{1}\right)$ is higher-order pseudo-invex at $u^{1}$ with respect to $\eta_{1}$ and $g^{1}\left(u^{1}, v^{1}, r^{1}\right)$,

(ii) $-f^{1}\left(x^{1},.\right)$ is higher-order pseudo-invex at $y^{1}$ with respect to $\eta_{2}$ and $-h^{1}\left(x^{1}, y^{1}, p^{1}\right)$,

(iii) $f^{2}\left(., v^{2}\right)$ is higher-order invex at $u^{2}$ with respect to $\eta_{3}$ and $g^{2}\left(u^{2}, v^{2}, r^{2}\right)$,

(iv) $-f^{2}\left(x^{2}\right.$,.) is higher-order invex at $y^{2}$ with respect to $\eta_{4}$ and $-h^{2}\left(x^{2}, y^{2}, p^{2}\right)$,
(v) $\eta_{1}\left(x^{1}, u^{1}\right)+u^{1}+r^{1} \in C_{1}$,
(vi) $\eta_{2}\left(v^{1}, y^{1}\right)+y^{1}+p^{1} \in C_{3}$,
(vii) $\eta_{3}\left(x^{2}, u^{2}\right)+u^{2}+r^{2} \in C_{2}$,
(viii) $\eta_{4}\left(v^{2}, y^{2}\right)+y^{2}+p^{2} \in C_{4}$.

Then

$L\left(x^{1}, x^{2}, y^{1}, y^{2}, p^{1}, p^{2}\right) \geqq M\left(u^{1}, u^{2}, v^{1}, v^{2}, r^{1}, r^{2}\right)$.

Proof: From hypothesis (vii), (viii) and equations (3.2) and (3.8), we get

$$
\begin{aligned}
& \eta_{3}\left(x^{2}, u^{2}\right)\left[\nabla_{x^{2}} f^{2}\left(u^{2}, v^{2}\right)+\nabla_{r^{2}} g_{2}\left(u^{2}, v^{2}, r^{2}\right)\right] \\
& +u^{2}\left[\nabla_{x^{2}} f^{2}\left(u^{2}, v^{2}\right)+\nabla_{r^{2}} g_{2}\left(u^{2}, v^{2}, r^{2}\right)\right] \\
& \quad \geqq-r^{2}\left[\nabla_{x^{2}} f^{2}\left(u^{2}, v^{2}\right)+\nabla_{r^{2}} g_{2}\left(u^{2}, v^{2}, r^{2}\right)\right], \\
& \eta_{4}\left(v^{2}, y^{2}\right)\left[\nabla_{y^{2}} f\left(x^{2}, y^{2}\right)+\nabla_{p^{2}} h_{2}\left(x^{2}, y^{2}, p^{2}\right)\right] \\
& +y^{2}\left[\nabla_{y^{2}} f\left(x^{2}, y^{2}\right)+\nabla_{p^{2}} h_{2}\left(x^{2}, y^{2}, p^{2}\right)\right] \\
& \geqq-p^{2}\left[\nabla_{y^{2}} f\left(x^{2}, y^{2}\right)+\nabla_{p^{2}} h_{2}\left(x^{2}, y^{2}, p^{2}\right)\right] .
\end{aligned}
$$


Which on using equations (3.5) and (3.11) implies that

$$
\begin{aligned}
& \eta_{3}\left(x^{2}, u^{2}\right)\left[\nabla_{x^{2}} f^{2}\left(u^{2}, v^{2}\right)+\nabla_{r^{2}} g_{2}\left(u^{2}, v^{2}, r^{2}\right)\right] \\
& +u^{2}\left[\nabla_{x^{2}} f^{2}\left(u^{2}, v^{2}\right)+\nabla_{r^{2}} g_{2}\left(u^{2}, v^{2}, r^{2}\right)\right] \geqq 0, \\
& \eta_{4}\left(v^{2}, y^{2}\right)\left[\nabla_{y^{2}} f\left(x^{2}, y^{2}\right)+\nabla_{p^{2}} h_{2}\left(x^{2}, y^{2}, p^{2}\right)\right] \\
& +y^{2}\left[\nabla_{y^{2}} f\left(x^{2}, y^{2}\right)+\nabla_{p^{2}} h_{2}\left(x^{2}, y^{2}, p^{2}\right)\right] \geqq 0 .
\end{aligned}
$$

Now from hypothesis (iii) and (iv), we have

$$
\begin{aligned}
& f^{2}\left(x^{2}, v^{2}\right)-f^{2}\left(u^{2}, v^{2}\right)-h^{2}\left(x^{2}, v^{2}, p^{2}\right) \\
& +\left(p^{2}\right)^{T} \nabla_{p^{2}} h^{2}\left(x^{2}, v^{2}, p^{2}\right)+h^{2}\left(u^{2}, v^{2}, p^{2}\right) \\
& -\left(p^{2}\right)^{T} \nabla_{p^{2}} h^{2}\left(u^{2}, v^{2}, p^{2}\right) \\
\unrhd_{3}\left(x^{2}, u^{2}\right)\left[\nabla_{x^{2}} f^{2}\left(u^{2}, v^{2}\right)+\nabla_{r^{2}} g_{2}\left(u^{2}, v^{2}, r^{2}\right)\right], & \\
\text { and } \quad & f^{2}\left(x^{2}, y^{2}\right)-f^{2}\left(x^{2}, v^{2}\right)-h^{2}\left(x^{2}, y^{2}, p^{2}\right) \\
& +\left(p^{2}\right)^{T} \nabla_{p^{2}} h^{2}\left(x^{2}, y^{2}, p^{2}\right)+h^{2}\left(x^{2}, v^{2}, p^{2}\right) \\
& -\left(p^{2}\right)^{T} \nabla_{p^{2}} h^{2}\left(x^{2}, v^{2}, p^{2}\right) \\
\geqq & \eta_{4}\left(v^{2}, y^{2}\right)\left[\nabla_{y^{2}} f\left(x^{2}, y^{2}\right)+\nabla_{p^{2}} h_{2}\left(x^{2}, y^{2}, p^{2}\right)\right],
\end{aligned}
$$

which along with equations (4.2) and (4.3), we obtain

$$
\begin{aligned}
& f^{2}\left(x^{2}, v^{2}\right)-f^{2}\left(u^{2}, v^{2}\right)-h^{2}\left(x^{2}, v^{2}, p^{2}\right) \\
& +\left(p^{2}\right)^{T} \nabla_{p^{2}} h^{2}\left(x^{2}, v^{2}, p^{2}\right)+h^{2}\left(u^{2}, v^{2}, p^{2}\right) \\
& -\left(p^{2}\right)^{T} \nabla_{p^{2}} h^{2}\left(u^{2}, v^{2}, p^{2}\right) \\
& \geqq-u^{2}\left[\nabla_{x^{2}} f^{2}\left(u^{2}, v^{2}\right)+\nabla_{r^{2}} g_{2}\left(u^{2}, v^{2}, r^{2}\right)\right], \\
& \quad f^{2}\left(x^{2}, y^{2}\right)-f^{2}\left(x^{2}, v^{2}\right)-h^{2}\left(x^{2}, y^{2}, p^{2}\right) \\
& \quad+\left(p^{2}\right)^{T} \nabla_{p^{2}} h^{2}\left(x^{2}, y^{2}, p^{2}\right)+h^{2}\left(x^{2}, v^{2}, p^{2}\right) \\
& \quad-\left(p^{2}\right)^{T} \nabla_{p^{2}} h^{2}\left(x^{2}, v^{2}, p^{2}\right) \\
& \geqq y^{2}\left[\nabla_{y^{2}} f\left(x^{2}, y^{2}\right)+\nabla_{p^{2}} h_{2}\left(x^{2}, y^{2}, p^{2}\right)\right] .
\end{aligned}
$$

Now adding the above two equation, we get

$$
\begin{aligned}
& f^{2}\left(x^{2}, y^{2}\right)-h^{2}\left(x^{2}, y^{2}, p^{2}\right)+\left(p^{2}\right)^{T} \nabla_{p^{2}} h^{2}\left(x^{2}, y^{2}, p^{2}\right) \\
& -y^{2}\left[\nabla_{y^{2}} f\left(x^{2}, y^{2}\right)+\nabla_{p^{2}} h_{2}\left(x^{2}, y^{2}, p^{2}\right)\right] \\
& \geqq f^{2}\left(u^{2}, v^{2}\right)-h^{2}\left(u^{2}, v^{2}, p^{2}\right)+\left(p^{2}\right)^{T} \nabla_{p^{2}} h^{2}\left(u^{2}, v^{2}, p^{2}\right) \\
& -u^{2}\left[\nabla_{x^{2}} f^{2}\left(u^{2}, v^{2}\right)+\nabla_{r^{2}} g_{2}\left(u^{2}, v^{2}, r^{2}\right)\right] .
\end{aligned}
$$

Similarly, from hypothesis (i), (ii) and equations (3.1), (3.7), we get

$$
\begin{aligned}
& \eta_{1}\left(x^{1}, u^{1}\right)\left[\nabla_{u^{1}} f_{1}\left(u^{1}, v^{1}\right)+\nabla_{r^{1}} g_{1}\left(u^{1}, v^{1}, r^{1}\right)\right] \\
& \geqq-\left(u^{1}+r^{1}\right)\left[\nabla_{u^{1}} f_{1}\left(u^{1}, v^{1}\right)+\nabla_{r^{1}} g_{1}\left(u^{1}, v^{1}, r^{1}\right)\right], \\
& \eta_{2}\left(v^{1}, y^{1}\right)\left[\nabla_{y^{1}} f\left(x^{1}, y^{1}\right)+\nabla_{p^{1}} h_{1}\left(x^{1}, y^{1}, p^{1}\right)\right] \\
& \geqq-\left(y^{1}+p^{1}\right)\left[\nabla_{y^{1}} f\left(x^{1}, y^{1}\right)+\nabla_{p^{1}} h_{1}\left(x^{1}, y^{1}, p^{1}\right)\right] .
\end{aligned}
$$

and

Now inequalities (3.3), (3.4), (3.9) and (3.10) gives

$$
\eta_{1}\left(x^{1}, u^{1}\right)\left[\nabla_{u^{1}} f_{1}\left(u^{1}, v^{1}\right)+\nabla_{r^{1}} g_{1}\left(u^{1}, v^{1}, r^{1}\right)\right] \geqq 0,
$$

and

$$
\eta_{2}\left(v^{1}, y^{1}\right)\left[\nabla_{y^{1}} f\left(x^{1}, y^{1}\right)+\nabla_{p^{1}} h_{1}\left(x^{1}, y^{1}, p^{1}\right)\right] \geqq 0,
$$

which by hypothesis (i) and (ii) implies

$$
\begin{aligned}
& f_{1}\left(x^{1}, v^{1}\right)-f_{1}\left(u^{1}, v^{1}\right)-h^{1}\left(x^{1}, v^{1}, p^{1}\right) \\
& +\left(p^{1}\right)^{T} \nabla_{p^{1}} h^{1}\left(x^{1}, v^{1}, p^{1}\right) \\
& +h^{1}\left(u^{1}, v^{1}, p^{1}\right)-\left(p^{1}\right)^{T} \nabla_{p^{1}} h^{1}\left(u^{1}, v^{1}, p^{1}\right) \geqq 0,
\end{aligned}
$$

and

$$
\begin{aligned}
& f_{1}\left(x^{1}, y^{1}\right)-f_{1}\left(x^{1}, v^{1}\right)-h^{1}\left(x^{1}, y^{1}, p^{1}\right) \\
& +\left(p^{1}\right)^{T} \nabla_{p} h^{1}\left(x^{1}, y^{1}, p^{1}\right) \\
& +h^{1}\left(x^{1}, v^{1}, p^{1}\right)-\left(p^{1}\right)^{T} \nabla_{p} h^{1}\left(x^{1}, v^{1}, p^{1}\right) \geqq 0 .
\end{aligned}
$$

Adding the above two inequalities, we get

$$
\begin{aligned}
& f_{1}\left(x^{1}, y^{1}\right)-h^{1}\left(x^{1}, y^{1}, p^{1}\right)+\left(p^{1}\right)^{T} \nabla_{p} h^{1}\left(x^{1}, y^{1}, p^{1}\right) \\
& \quad \geqq f_{1}\left(u^{1}, v^{1}\right)-h^{1}\left(u^{1}, v^{1}, p^{1}\right)+\left(p^{1}\right)^{T} \nabla_{p} h^{1}\left(u^{1}, v^{1}, p^{1}\right) .
\end{aligned}
$$

Combining inequalities (4.4) and (4.5), we have

$$
L\left(x^{1}, x^{2}, y^{1}, y^{2}, p^{1}, p^{2}\right) \geqq M\left(u^{1}, u^{2}, v^{1}, v^{2}, r^{1}, r^{2}\right) .
$$

Thus the results holds.

Theorem 4.2 (Strong Duality).

Let $\left(\bar{x}^{1}, \bar{x}^{2}, \bar{y}^{1}, \bar{y}^{2}, \bar{p}^{1}, \bar{p}^{2}\right)$ be an optimal solution of (MHPC). Suppose that

(i) $\nabla_{p^{1}{ }^{1}} h^{1}\left(\bar{x}^{1}, \bar{y}^{1}, \bar{p}^{1}\right)$ is positive or negative definite and $\nabla_{p^{2} p^{2}} h^{2}\left(\bar{x}^{2}, \bar{y}^{2}, \bar{p}^{2}\right)$ is negative definite,

(ii)

$$
\nabla_{y}^{1} f^{1}\left(\bar{x}^{1}, \bar{y}^{1}\right)+\nabla_{p}^{1} h^{1}\left(\bar{x}^{1}, \bar{y}^{1}, \bar{p}^{1}\right) \neq 0
$$

$$
\nabla_{y}^{2} f^{2}\left(\bar{x}^{2}, \bar{y}^{2}\right)+\nabla_{p}^{2} h^{2}\left(\bar{x}^{2}, \bar{y}^{2}, \bar{p}^{2}\right) \neq 0,
$$

$\left(\bar{p}^{1}\right)^{T}\left[\nabla_{y}^{1} f^{1}\left(\bar{x}^{1}, \bar{y}^{1}\right)+\nabla_{p}^{1} h^{1}\left(\bar{x}^{1}, \bar{y}^{1}, \bar{p}^{1}\right)\right]=0 \Rightarrow \bar{p}^{1}=0$ and 


$$
\begin{gathered}
y^{2}\left[\nabla_{y^{2}} h^{2}\left(\bar{x}^{2}, \bar{y}^{2}, \bar{p}^{2}\right)-\nabla_{p^{2}} h^{2}\left(\bar{x}^{2}, \bar{y}^{2}, \bar{p}^{2}\right)\right. \\
\left.+\nabla_{y^{2} y^{2}} f^{2}\left(\bar{x}^{2}, \bar{y}^{2}\right) \bar{p}^{2}\right]=0 \Rightarrow \bar{p}^{2}=0, \\
\left(\text { iv) } h^{1}\left(\bar{x}^{1}, \bar{y}^{1}, 0\right)=g^{1}\left(\bar{x}^{1}, \bar{y}^{1}, 0\right),\right. \\
\nabla_{x^{1}} h^{1}\left(\bar{x}^{1}, \bar{y}^{1}, 0\right)=\nabla_{{ }^{1}} g^{1}\left(\bar{x}^{1}, \bar{y}^{1}, 0\right), \\
\nabla_{y^{1}} h^{1}\left(\bar{x}^{1}, \bar{y}^{1}, 0\right)=\nabla_{p^{1}} h^{1}\left(\bar{x}^{1}, \bar{y}^{1}, 0\right) \text { and } \\
h^{2}\left(\bar{x}^{2}, \bar{y}^{2}, 0\right)=g^{2}\left(\bar{x}^{2}, \bar{y}^{2}, 0\right), \\
\nabla_{x^{2}} h^{2}\left(\bar{x}^{2}, \bar{y}^{2}, 0\right)=\nabla_{r^{2}} g^{2}\left(\bar{x}^{2}, \bar{y}^{2}, 0\right) .
\end{gathered}
$$

Then

(I) $\left(\bar{x}^{1}, \bar{x}^{2}, \bar{y}^{1}, \bar{y}^{2}, \bar{r}^{1}=0, \bar{r}^{2}=0\right)$ is feasible for (MHDC) and

(II)

$$
L\left(\bar{x}^{1}, \bar{x}^{2}, \bar{y}^{1}, \bar{y}^{2}, \bar{p}^{1}, \bar{p}^{2}\right)=M\left(\bar{x}^{1}, \bar{x}^{2}, \bar{y}^{1}, \bar{y}^{2}, \bar{r}^{1}, \bar{r}^{2}\right) .
$$

Furthermore, if the hypothesis of Theorem (4.1) are satisfied for all feasible solutions of (MHPC) and (MHDC), then $\left(\bar{x}^{1}, \bar{x}^{2}, \bar{y}^{1}, \bar{y}^{2}, \bar{r}^{1}=0, \bar{r}^{2}=0\right) \quad$ is $\quad$ an optimal solution for (MHDC).

Proof: Since $\left(\bar{x}^{1}, \bar{x}^{2}, \bar{y}^{1}, \bar{y}^{2}, \bar{p}^{1}, \bar{p}^{2}\right)$ is a n optimal solution of (MHPC), by the Fritz John necessary optimality conditions [1], there exist $\alpha, \gamma, \delta^{1}, \delta^{2} \in R, \beta^{1} \in C_{3}$, $\beta^{2} \in C_{4}$, and $\mu^{1} \in C_{1}, \mu^{2} \in C_{2}$ such that the following conditions are satisfied at $\left(\bar{x}^{1}, \bar{x}^{2}, \bar{y}^{1}, \bar{y}^{2}, \bar{p}^{1}, \bar{p}^{2}\right)$ :

$$
\begin{aligned}
& {\left[\alpha \left[\nabla_{x^{1}} f^{1}\left(\bar{x}^{1}, \bar{y}^{1}\right)+\nabla_{x^{1}} h^{1}\left(\bar{x}^{1}, \bar{y}^{1}, \bar{p}^{1}\right)\right.\right.} \\
& \left.-\nabla_{p^{1} x^{1}} h^{1}\left(\bar{x}^{1}, \bar{y}^{1}, \bar{p}^{1}\right) \bar{p}^{1}\right]+\left[\nabla_{y^{1} x^{1}} f^{1}\left(\bar{x}^{1}, \bar{y}^{1}\right)\right.
\end{aligned}
$$

$\left.\left.+\nabla_{p^{1} x^{1}} h^{1}\left(\bar{x}^{1}, \bar{y}^{1}, \bar{p}^{1}\right)\right]\left(\beta^{1}-\gamma \bar{y}^{1}-\delta^{1} \bar{p}^{1}\right)-\mu^{1}\right]\left(x^{1}-\bar{x}^{1}\right)$ $\geqq 0$, forall, $x^{1} \in C_{1}$,

$$
\begin{aligned}
& \alpha\left[\nabla_{x^{2}} f^{2}\left(\bar{x}^{2}, \bar{y}^{2}\right)+\nabla_{x^{2}} h^{2}\left(\bar{x}^{2}, \bar{y}^{2}, \bar{p}^{2}\right)\right] \\
& +\left\{\nabla_{p^{2} x^{2}} h^{2}\left(\bar{x}^{2}, \bar{y}^{2}, \bar{p}^{2}\right)\right\}\left(\beta^{2}-\alpha^{2} \bar{y}^{2}-\alpha^{2} \bar{p}^{2}-\delta^{2} \bar{p}^{2}\right) \\
& +\left\{\nabla_{y^{2} x^{2}} f^{2}\left(\bar{x}^{2}, \bar{y}^{2}\right)\right\}\left(\beta^{2}-\alpha^{2} \bar{y}^{2}-\delta^{2} \bar{p}^{2}\right) \\
& \left.-\mu^{2}\right]\left(x^{2}-\bar{x}^{2}\right) \geqq 0, \text { forall, } x^{2} \in C_{2}, \\
& \alpha\left[\nabla_{y}^{1} f^{1}\left(\bar{x}^{1}, \bar{y}^{1}\right)+\nabla_{y}^{1} h^{1}\left(\bar{x}^{1}, \bar{y}^{1}, \bar{p}^{1}\right)\right. \\
& \left.-\nabla_{p^{1} y^{1}} h^{1}\left(\bar{x}^{1}, \bar{y}^{1}, \bar{p}^{1}\right) \bar{p}^{1}\right]+\left(\nabla_{y^{1}{ }^{1}} f^{1}\left(\bar{x}^{1}, \bar{y}^{1}\right)\right. \\
& \left.+\nabla_{p^{1}{ }^{1}} h^{1}\left(\bar{x}^{1}, \bar{y}^{1}, \bar{p}^{1}\right)\right)\left(\beta^{1}-\gamma \bar{y}^{1}-\delta^{1} \bar{p}^{1}\right) \\
& -\gamma\left[\nabla_{y}^{1} f^{1}\left(\bar{x}^{1}, \bar{y}^{1}\right)+\nabla_{p}^{1} h^{1}\left(\bar{x}^{1}, \bar{y}^{1}, \bar{p}^{1}\right)\right] \geqq 0, \\
& \text { forall, } y^{1} \in R^{\left|K_{1}\right|},
\end{aligned}
$$

$$
\begin{aligned}
& \left\{\nabla_{p^{2} y^{2}} h^{2}\left(\bar{x}^{2}, \bar{y}^{2}, \bar{p}^{2}\right)\right\}\left(\beta^{2}-\alpha^{2} \bar{y}^{2}-\alpha^{2} \bar{p}^{2}-\delta^{2} \bar{p}^{2}\right) \\
& +\alpha^{2}\left[\nabla_{y^{2}} h^{2}\left(\bar{x}^{2}, \bar{y}^{2}, \bar{p}^{2}\right)-\nabla_{p^{2}} h^{2}\left(\bar{x}^{2}, \bar{y}^{2}, \bar{p}^{2}\right)\right] \\
& \quad+\left\{\nabla_{y^{2} y^{2}} f^{2}\left(\bar{x}^{2}, \bar{y}^{2}\right)\right\}\left(\beta^{2}-\alpha^{2} \bar{y}^{2}-\delta^{2} \bar{p}^{2}\right) \\
& \quad-\xi^{2} \geqq 0, \text { forall, } y^{2} \in R^{\left|K_{2}\right|}
\end{aligned}
$$

$\left\{\nabla_{p^{1} p^{1}} h^{1}\left(\bar{x}^{1}, \bar{y}^{1}, \bar{p}^{1}\right)\right\}\left(\beta^{1}-\alpha \bar{p}^{1}-\gamma \bar{y}^{1}-\delta^{1} \bar{p}^{1}\right)$

$-\delta^{1}\left[\nabla_{y}^{1} f^{1}\left(\bar{x}^{1}, \bar{y}^{1}\right)+\nabla_{p}^{1} h^{1}\left(\bar{x}^{1}, \bar{y}^{1}, \bar{p}^{1}\right)\right]=0$,

$\left\{\nabla_{p^{2} p^{2}} h^{2}\left(\bar{x}^{2}, \bar{y}^{2}, \bar{p}^{2}\right)\right\}\left(\beta^{2}-\alpha \bar{y}^{2}-\alpha \bar{p}^{2}-\delta^{2} \bar{p}^{2}\right)$

$-\delta^{2}\left[\nabla_{y^{2}} f^{2}\left(\bar{x}^{2}, \bar{y}^{2}\right)+\nabla_{p^{2}} h^{2}\left(\bar{x}^{2}, \bar{y}^{2}, \bar{p}^{2}\right)\right]=0$,

$$
\beta^{1}\left[\nabla_{y^{1}} f^{1}\left(\bar{x}^{1}, \bar{y}^{1}\right)+\nabla_{p}^{1} h^{1}\left(\bar{x}^{1}, \bar{y}^{1}, \bar{p}^{1}\right)\right]=0,
$$

$$
\beta^{2}\left[\nabla_{y^{2}} f^{2}\left(\bar{x}^{2}, \bar{y}^{2}\right)+\nabla_{p^{2}} h^{2}\left(\bar{x}^{2}, \bar{y}^{2}, \bar{p}^{2}\right)\right]=0,
$$

$$
\bar{y}^{1}\left[\nabla_{y^{1}} f^{1}\left(\bar{x}^{1}, \bar{y}^{1}\right)+\nabla_{p}^{1} h^{1}\left(\bar{x}^{1}, \bar{y}^{1}, \bar{p}^{1}\right)\right]=0,
$$

$\delta^{1} \bar{p}^{1}\left[\nabla_{y^{1}} f^{1}\left(\bar{x}^{1}, \bar{y}^{1}\right)+\nabla_{p}^{1} h^{1}\left(\bar{x}^{1}, \bar{y}^{1}, \bar{p}^{1}\right)\right]=0$,

$$
\delta^{2} \bar{p}^{2}\left[\nabla_{y^{2}} f^{2}\left(\bar{x}^{2}, \bar{y}^{2}\right)+\nabla_{p^{2}} h^{2}\left(\bar{x}^{2}, \bar{y}^{2}, \bar{p}^{2}\right)\right]=0,
$$

$$
\begin{aligned}
& \mu^{1} \bar{x}^{1}=0, \\
& \mu^{2} \bar{x}^{2}=0, \\
& \xi^{2} \bar{y}^{2}=0,
\end{aligned}
$$

$$
\begin{array}{r}
\left(\alpha, \beta^{1}, \beta^{2}, \gamma, \delta^{1}, \delta^{2}, \mu^{1}, \mu^{2}, \xi^{2}\right) \neq 0, \\
\left(\alpha, \beta^{1}, \beta^{2}, \gamma, \delta^{1}, \delta^{2}, \mu^{1}, \mu^{2}, \xi^{2}\right) \geqq 0,
\end{array}
$$

Premultiplying equations (4.10), (4.11) by $\left(\beta^{1}-\alpha \bar{p}^{1}-\bar{y}^{1}-\delta^{1} \bar{p}^{1}\right),\left(\beta^{2}-\alpha \bar{p}^{2}-\alpha \bar{y}^{2}-\delta^{2} \bar{p}^{2}\right)$, respectively and then using equations (4.12)-(4.16), we get

$$
\begin{aligned}
& \left(\beta^{1}-\alpha \bar{p}^{1}-\gamma \bar{y}^{1}-\delta^{1} \bar{p}^{1}\right)^{T} \\
& \nabla_{p^{1} p^{1}} h\left(\bar{x}^{1},\left(^{4},{ }^{1}, \bar{p}^{1}\right)\left(\beta^{1}-\alpha \bar{p}^{1}-\gamma \bar{y}^{1}-\delta^{1} \bar{p}^{1}\right)=0,\right.
\end{aligned}
$$

And

$$
\begin{aligned}
& \left(\beta^{2}-\alpha \bar{p}^{2}-\alpha \bar{y}^{2}-\delta^{2} \bar{p}^{2}\right) \nabla_{p^{2} p^{2}} h\left(\bar{x}^{2}, \bar{y}^{2}, \bar{p}^{2}\right) \\
& \left(\beta^{2}-\alpha \bar{p}^{2}-\alpha \bar{y}^{2}-\delta^{2} \bar{p}^{2}\right) \\
& \quad=-\alpha \delta \bar{y}^{2}\left[\nabla_{y^{2}} f^{2}\left(\bar{x}^{2}, \bar{y}^{2}\right)+\nabla_{p^{2}} h^{2}\left(\bar{x}^{2}, \bar{y}^{2}, \bar{p}^{2}\right)\right] .
\end{aligned}
$$

Using hypothesis (i), we get

$$
\beta^{1}=\alpha \bar{p}^{1}+\gamma \bar{y}^{1}+\delta^{1} \bar{p}^{1} .
$$

Further using inequality (3.1), (3.6) and (4.21), we obtain

$$
\begin{aligned}
& \left(\beta^{2}-\alpha \bar{p}^{2}-\alpha \bar{y}^{2}-\delta^{2} \bar{p}^{2}\right) \nabla_{p^{2}{ }^{2}} h\left(\bar{x}^{2}, \bar{y}^{2}, \bar{p}^{2}\right) \\
& \left(\beta^{2}-\alpha \bar{p}^{2}-\alpha \bar{y}^{2}-\delta^{2} \bar{p}^{2}\right) \geqq 0,
\end{aligned}
$$


which on using hypothesis (i)

$$
\beta^{2}=\alpha \bar{p}^{2}+\alpha \bar{y}^{2}+\delta^{2} \bar{p}^{2}
$$

From equations (4.10) and (4.11), and hypothesis (ii), we obtain

$$
\delta^{1}=0
$$

And

$$
\delta^{2}=0
$$

Now suppose, $\alpha=0$. Then equations (4.23), with (4.9) gives $\xi^{2}=0$ and, with (4.25) implies $\beta^{2}=0$ also equations (4.6), (4.22) implies $\mu^{1}=0$ and equations (4.7), (4.23) and (4.25) implies $\mu^{2}=0$. From equation (4.8) and hypothesis (ii) yield $\gamma=0$, which along with equation (4.22), (4.24) reduces $\beta^{1}=0 \quad$. Thus $\left(\alpha, \beta^{1}, \beta^{2}, \gamma, \delta^{1}, \delta^{2}, \mu^{1}, \mu^{2}\right)=0$, a contradiction to (4.20).

$$
\text { Hence } \alpha=0 \text {. }
$$

Using equations (4.12), (4.14) and (4.15), we have

$$
\begin{aligned}
& \left(\beta^{1}-\gamma \bar{y}^{1}-\delta^{1} \bar{p}^{1}\right)^{T}\left[\nabla_{y}^{1} f^{1}\left(\bar{x}^{1}, \bar{y}^{1}\right)\right. \\
& \left.+\nabla_{p} h\left(\bar{x}^{1}, \bar{y}^{1}, \bar{p}^{1}\right)\right]=0,
\end{aligned}
$$

Now equation (4.22), gives

$$
\alpha \bar{p}^{1^{T}}\left[\nabla_{y}^{1} f^{1}\left(\bar{x}^{1}, \bar{y}^{1}\right)+\nabla_{p} h\left(\bar{x}^{1}, \bar{y}^{1}, \bar{p}^{1}\right)\right]=0 .
$$

which along with hypothesis (iii) yield

$$
\bar{p}^{1}=0
$$

Further, from equation (4.9) and (4.23), we get

$$
\begin{aligned}
& \alpha^{2}\left[\nabla_{y^{2}} h^{2}\left(\bar{x}^{2}, \bar{y}^{2}, \bar{p}^{2}\right)-\nabla_{p^{2}} h^{2}\left(\bar{x}^{2}, \bar{y}^{2}, \bar{p}^{2}\right)\right. \\
& \left.+\nabla_{y^{2} y^{2}} f^{2}\left(\bar{x}^{2}, \bar{y}^{2}\right) \bar{p}^{2}\right]-\xi^{2}=0 .
\end{aligned}
$$

Now from hypothesis (iii), we obtain

$$
\bar{p}^{2}=0
$$

Therefore equation (4.22) and (4.23) reduce to

$$
\beta^{1}=\bar{y}^{1}
$$

And

$$
\beta^{2}=\alpha \bar{y}^{2}
$$

Also, it follows from equations (4.8), (4.22), (4.28) and hypothesis (ii) and (iv) that

$$
\alpha=\gamma>0
$$

So equation (4.31) implies

$$
\bar{y}^{1}=\frac{\beta^{1}}{\gamma} \oplus .
$$

Moreover, equation (4.6), (4.7) along with (4.22), (4.23), (4.30) and hypothesis (iv) yields

$$
\begin{aligned}
& \alpha\left[\nabla_{x_{1}} f^{1}\left(\bar{x}^{1}, \bar{y}^{1}\right)+\nabla_{r^{1}} h^{1}\left(\bar{x}^{1}, \bar{y}^{1}, \bar{p}^{1}\right)\right. \\
& \left.-\mu^{1}\right]\left(x^{1}-\bar{x}^{1}\right) \geqq 0, \text { forall } x^{1} \in C_{1}, \\
& \alpha\left[\nabla_{x^{2}} f^{2}\left(\bar{x}^{2}, \bar{y}^{2}\right)+\nabla_{r^{2}} h^{2}\left(\bar{x}^{2}, \bar{y}^{2}, \bar{p}^{2}\right)\right. \\
& \left.-\mu^{2}\right]\left(x^{2}-\bar{x}^{2}\right) \geqq 0, \text { forall } x^{2} \in C_{2},
\end{aligned}
$$

Let $x^{1} \in C_{1}$, then $\bar{x}^{1}+x^{1} \in C_{1}$ and then above inequality implies

$$
\begin{aligned}
& \alpha x^{1 T}\left[\nabla_{x^{2}} f^{2}\left(\bar{x}^{2}, \bar{y}^{2}\right)+\nabla_{r^{2}} h^{2}\left(\bar{x}^{2}, \bar{y}^{2}, \bar{p}^{2}\right)\right. \\
& \left.-\mu^{2}\right] \geqq 0, \text { forall } x^{1} \in C_{1},
\end{aligned}
$$

Further by using equation (4.17) the above inequality also be rewritten as

$$
\begin{aligned}
& \bar{x}^{1 T}\left[\nabla_{x^{1}} f^{1}\left(\bar{x}^{1}, \bar{y}^{1}\right)+\nabla_{r} h^{1}\left(\bar{x}^{1}, \bar{y}^{1}, \bar{p}^{1}\right)\right] \\
& \geqq \mu^{1} \bar{x}^{1}=0, \text { forall } x^{1} \in C_{1} .
\end{aligned}
$$

Therefore

$$
\nabla_{x^{1}} f^{1}\left(\bar{x}^{1}, \bar{y}^{1}\right)+\nabla_{{ }_{1}} h^{1}\left(\bar{x}^{1}, \bar{y}^{1}, \bar{p}^{1}\right) \in C_{1}^{*},
$$

Similarly, we also obtain that

$$
\nabla_{x^{2}} f^{2}\left(\bar{x}^{2}, \bar{y}^{2}\right)+\nabla_{r^{2}} h^{2}\left(\bar{x}^{2}, \bar{y}^{2}, \bar{p}^{2}\right) \in C_{2}{ }^{*} \text {. }
$$

Thus $\left(\bar{x}^{1}, \bar{x}^{2}, \bar{y}^{1}, \bar{y}^{2}, \bar{r}^{1}=0, \bar{r}^{2}=0\right)$ satisfies the dual constraints (3.7)-(3.12), i.e, it is an feasible solution of (MHDC).

Also, using hypothesis (iv) we get the values of the objective functions of (MHPC) and (MHDC) at $\left(\bar{x}^{1}, \bar{x}^{2}, \bar{y}^{1}, \bar{y}^{2}, \bar{p}^{1}=0, \bar{p}^{2}=0\right)$ and $\left(\bar{x}^{1}, \bar{x}^{2}, \bar{y}^{1}, \bar{y}^{2}, \bar{r}^{1}=0, \bar{r}^{2}=0\right)$ are equal. Using Weak duality it easily shown that 
$\left(\bar{x}^{1}, \bar{x}^{2}, \bar{y}^{1}, \bar{y}^{2}, \bar{r}^{1}=0, \bar{r}^{2}=0\right)$

and

$\left(\bar{x}^{1}, \bar{x}^{2}, \bar{y}^{1}, \bar{y}^{2}, \bar{p}^{1}=0, \bar{p}^{2}=0\right)$ are an optimal solutions for $(M H P C)$ and $(M H D C)$, respectively.

Theorem 4.3 (Converse Duality).

Let $\left(\bar{u}^{1}, \bar{u}^{2}, \bar{v}^{1}, \bar{v}^{2}, \bar{r}^{1}, \bar{r}^{2}\right)$ be an optimal solution of $(M H D C)$. Suppose that

(i) $\nabla_{r^{1} r^{1}} g^{1}\left(\bar{u}^{1}, \bar{v}^{1}, \bar{r}^{1}\right)$ is positive or negative definite and $\nabla_{r^{2} r^{2}} g^{2}\left(\bar{u}^{2}, \bar{v}^{2}, \bar{r}^{2}\right)$ is negative definite,

(ii) $\nabla_{u}^{1} f^{1}\left(\bar{u}^{1}, \bar{v}^{1}\right)+\nabla_{r}^{1} g^{1}\left(\bar{u}^{1}, \bar{v}^{1}, \bar{r}^{1}\right) \neq 0$ and $\nabla_{y}^{2} f^{2}\left(\bar{x}^{2}, \bar{y}^{2}\right)+\nabla_{p}^{2} h^{2}\left(\bar{x}^{2}, \bar{y}^{2}, \bar{p}^{2}\right) \neq 0$,

(iii)

$\left(\bar{r}^{1}\right)^{T}\left[\nabla_{u}^{1} f^{1}\left(\bar{u}^{1}, \bar{v}^{1}\right)+\nabla_{r}^{1} g^{1}\left(\bar{u}^{1}, \bar{v}^{1}, \bar{r}^{1}\right)\right]=0 \Rightarrow \bar{r}^{1}=0$ and

$$
\begin{aligned}
& \nabla_{u^{2}} g^{2}\left(\bar{u}^{2}, \bar{v}^{2}, \bar{r}^{2}\right)-\nabla_{r^{2}} g^{2}\left(\bar{u}^{2}, \bar{v}^{2}, \bar{r}^{2}\right) \\
& +\nabla_{u^{2} u^{2}} f^{2}\left(\bar{u}^{2}, \bar{v}^{2}\right) \bar{r}^{2}=0 \Rightarrow \bar{r}^{2}=0,
\end{aligned}
$$

$$
g^{1}\left(\bar{u}^{1}, \bar{v}^{1}, 0\right)=g^{1}\left(\bar{u}^{1}, \bar{v}^{1}, 0\right),
$$

$\nabla_{u^{1}} g^{1}\left(\bar{u}^{1}, \bar{v}^{1}, 0\right)=\nabla_{r^{1}} g^{1}\left(\bar{u}^{1}, \bar{v}^{1}, 0\right)$,

$\nabla_{v^{1}} g^{1}\left(\bar{u}^{1}, \bar{v}^{1}, 0\right)=\nabla_{r^{1}} g^{1}\left(\bar{u}^{1}, \bar{v}^{1}, 0\right) \quad$ and $g^{2}\left(\bar{u}^{2}, \bar{v}^{2}, 0\right)=h^{2}\left(\bar{u}^{2}, \bar{v}^{2}, 0\right)$,

$$
\nabla_{u^{2}} g^{2}\left(\bar{u}^{2}, \bar{v}^{2}, 0\right)=\nabla_{r^{2}} h^{2}\left(\bar{u}^{2}, \bar{v}^{2}, 0\right) .
$$

Then

(I) $\left(\bar{u}^{1}, \bar{u}^{2}, \bar{v}^{1}, \bar{v}^{2}, \bar{r}^{1}=0, \bar{r}^{2}=0\right)$ is feasible for $(M H P C)$ and

(II)

$L\left(\bar{u}^{1}, \bar{u}^{2}, \bar{v}^{1}, \bar{v}^{2}, \bar{p}^{1}, \bar{p}^{2}\right)=M\left(\bar{u}^{1}, \bar{u}^{2}, \bar{v}^{1}, \bar{v}^{2}, \bar{r}^{1}, \bar{r}^{2}\right)$.

Furthermore, if the hypothesis of Theorem 4.1 are satisfied for all feasible solutions of (MHPC) and (MHDC), then $\left(\bar{x}^{1}, \bar{x}^{2}, \bar{y}^{1}, \bar{y}^{2}, \bar{r}^{1}=0, \bar{r}^{2}=0\right)$ is an optimal solution for (MHPC).

Proof. Follows on the line of Theorem 4.2.

Theorem 4.4 (Self Duality).

A primal (dual) problem having equivalent dual (primal) formulation is said to be self-dual, that is, if the dual can be recast in the form of the primal. In general, (MHPC) and $(M H D C)$ are not self-duals without some added restrictions on $\mathrm{f} ; \mathrm{g} ;$ and $\mathrm{h}$. If we assume $f^{1}: R^{\left|J_{1}\right|} \times R^{\left|K_{1}\right|} \rightarrow R, \quad f^{2}: R^{\left|J_{2}\right|} \times R^{\left|K_{2}\right|} \rightarrow R$, $g^{1}: R^{\left|J_{1}\right|} \times R^{\left|K_{1}\right|} \rightarrow R, \quad g^{2}: R^{\left|J_{2}\right|} \times R^{\left|K_{2}\right|} \rightarrow R$, $h^{1}: R^{\left|J_{1}\right|} \times R^{\left|K_{1}\right|} \times R^{\left|K_{1}\right|} \rightarrow R$,

$h^{2}: R^{\left|J_{2}\right|} \times R^{\left|K_{2}\right|} \times R^{\left|K_{2}\right|} \rightarrow R$, to be skew symmetric, that is

$$
f^{i}\left(u^{1}, v^{1}\right)=-f^{i}\left(u^{1}, v^{1}\right), i=1,2 .
$$

and

$$
g^{i}\left(u^{1}, v^{1}, r^{1}\right)=-g^{i}\left(u^{1}, v^{1}, r^{1}\right), i=1,2,
$$

Then we shall show that $(M H P C)$ and $(M H D C)$ are self-duals. By recasting the dual problem (MHDC) as a minimization problem, we have

Minimize $M(u, v, r)=$

$$
\begin{gathered}
-\left\{f_{1}\left(u^{1}, v^{1}\right)+f_{2}\left(u^{2}, v^{2}\right)+g_{1}\left(u^{1}, v^{1}, r^{1}\right)+g_{2}\left(u^{2}, v^{2}, r^{2}\right)\right. \\
-\left(r^{1}\right)^{T} \nabla_{r^{1}} g_{1}\left(u^{1}, v^{1}, r^{1}\right)-\left(r^{2}\right)^{T} \nabla_{r^{2}} g_{2}\left(u^{2}, v^{2}, r^{2}\right) \\
\left.-\left(u^{2}\right)^{T}\left[\nabla_{u^{2}} f^{2}\left(u^{2}, v^{2}\right)+\nabla_{r^{2}} g_{2}\left(u^{2}, v^{2}, r^{2}\right)\right]\right\}
\end{gathered}
$$

Subject to

$$
\begin{gathered}
\nabla_{u^{1}} f_{1}\left(u^{1}, v^{1}\right)+\nabla_{r^{1}} g_{1}\left(u^{1}, v^{1}, r^{1}\right) \geqq 0, \\
\nabla_{u^{2}} f^{2}\left(u^{2}, v^{2}\right)+\nabla_{r^{2}} g_{2}\left(u^{2}, v^{2}, r^{2}\right) \geqq 0, \\
\left(u^{1}\right)^{T}\left[\nabla_{u^{1}} f_{1}\left(u^{1}, v^{1}\right)+\nabla_{r^{1}} g_{1}\left(u^{1}, v^{1}, r^{1}\right)\right] \leqq 0, \\
\left(r^{1}\right)^{T}\left[\nabla_{u^{1}} f_{1}\left(u^{1}, v^{1}\right)+\nabla_{r^{1}} g_{1}\left(u^{1}, v^{1}, r^{1}\right)\right] \leqq 0, \\
\left(r^{2}\right)^{T}\left[\nabla_{u^{2}} f^{2}\left(u^{2}, v^{2}\right)+\nabla_{r^{2}} g_{2}\left(u^{2}, v^{2}, r^{2}\right)\right] \leqq 0, \\
v^{1}, v^{2}, u^{2} \geqq 0,
\end{gathered}
$$

As $\mathrm{f}, \mathrm{g}$ and $\mathrm{h}$ are skew symmetric, i.e.,

$$
\begin{aligned}
& \nabla_{u^{1}} f_{1}\left(u^{1}, v^{1}\right)=-\nabla_{u^{1}} f_{1}\left(v^{1}, u^{1}\right), \\
& \nabla_{u^{1}} f_{2}\left(u^{2}, v^{2}\right)=-\nabla_{u^{1}} f_{2}\left(v^{2}, u^{2}\right), \\
& \nabla_{u^{1}} g_{1}\left(u^{1}, v^{1}, r^{1}\right)=-\nabla_{u^{1}} g_{1}\left(v^{1}, u^{1}, r^{1}\right), \text { and } \\
& \nabla_{u^{1}} g_{2}\left(u^{2}, v^{2}, r^{2}\right)=-\nabla_{u^{1}} g_{2}\left(v^{2}, u^{2}, r^{2}\right),
\end{aligned}
$$

Then the above problem becomes :

Minimize $M(u, v, r)=$

$$
\begin{gathered}
f_{1}\left(v^{1}, u^{1}\right)+f_{2}\left(v^{2}, u^{2}\right)+g_{1}\left(v^{1}, u^{1}, r^{1}\right)+g_{2}\left(v^{2}, u^{2}, r^{2}\right) \\
-\left(r^{1}\right)^{T} \nabla_{r^{1}} g_{1}\left(v^{1}, u^{1}, r^{1}\right)-\left(r^{2}\right)^{T} \nabla_{r^{2}} g_{2}\left(v^{2}, u^{2}, r^{2}\right) \\
-\left(u^{2}\right)^{T}\left[\nabla_{u^{2}} f^{2}\left(v^{2}, u^{2}\right)+\nabla_{r^{2}} g_{2}\left(v^{2}, u^{2}, r^{2}\right)\right]
\end{gathered}
$$

Subject to

$$
\begin{gathered}
\nabla_{u^{1}} f_{1}\left(v^{1}, u^{1}\right)+\nabla_{r^{1}} g_{1}\left(v^{1}, u^{1}, r^{1}\right) \leqq 0, \\
\nabla_{u^{2}} f^{2}\left(v^{2}, u^{2}\right)+\nabla_{r^{2}} g_{2}\left(v^{2}, u^{2}, r^{2}\right) \leqq 0, \\
\left(u^{1}\right)^{T}\left[\nabla_{u^{1}} f_{1}\left(v^{1}, u^{1}\right)+\nabla_{r^{1}} g_{1}\left(v^{1}, u^{1}, r^{1}\right)\right] \geqq 0, \\
\left(r^{1}\right)^{T}\left[\nabla_{u^{1}} f_{1}\left(v^{1}, u^{1}\right)+\nabla_{r^{1}} g_{1}\left(v^{1}, u^{1}, r^{1}\right)\right] \geqq 0, \\
\left(r^{2}\right)^{T}\left[\nabla_{u^{2}} f^{2}\left(v^{2}, u^{2}\right)+\nabla_{r^{2}} g_{2}\left(v^{2}, u^{2}, r^{2}\right)\right] \geqq 0, \\
v^{1}, v^{2}, u^{2} \geqq 0,
\end{gathered}
$$


Which shows that $M(u, v, p)$ is identical to $L(x, y, p)$, that is, the objective and the constraint functions are identical. Thus, the problem $L(x, y, p)$ becomes self-dual.

It is obvious that the feasibility of $\left(x^{1}, x^{2}, y^{1}, y^{2}, p^{1}, p^{2}\right)$ for $L(x, y, p)$ implies the feasibility of $\left(y^{1}, y^{2}, x^{1}, x^{2}, p^{1}, p^{2}\right)$ for $(M H P C)$ implies the feasibility of for (MHDC) and vice versa.

\section{SPeCIAL CASE}

In this section, we consider some special cases of our problems by choosing particular forms of the closed convex sets $C_{1}$ and $C_{2}$. In all these cases, $h(x, y, p)=(1 / 2) p^{T} \nabla_{y y} f(x, y) p$ and $g(u, v, r)=(1 / 2) r^{T} \nabla_{x x} f(u, v) r$,

(a) If $\left|K_{1}\right|=0,\left|J_{1}\right|=0, p=0$ and $r=0$, then $(M H P C)$ and $(M H D C)$ reduce to the programs studied in Chandra and Kumar [14].

(b) If $\left|K_{1}\right|=0,\left|J_{1}\right|=0, C_{1}=R_{+}^{n}$ and $C_{2}=R_{+}^{m}$, then after removing inequalities (3.5), (3.11), our programs reduce to the problems considered in Mishra [4].

(c) If $\left|K_{1}\right|=0,\left|J_{1}\right|=0, C_{1}=R_{+}^{n}$ and $C_{2}=R_{+}^{m}$, then the then after removing inequalities (3.5), (3.11), programs reduce to the second-order symmetric dual programs of Gulati et al. [15].

\section{CONCLUSION}

A pair of mixed symmetric dual programs has been formulated by considering the optimization under the assumptions of $\eta$-invexity and $\eta$-pseudoinvexity. It may be noted that the symmetric duality between $(M H P C)$ and (MHDC) can be utilized to establish non differentiable mixed symmetric duality in integer over cone and other related programming problems.

\section{ACKNOWLEDGMENT}

The research of the author Khushboo Verma is supported by the Department of Atomic Energy, Govt. of India, under the NBHM Post-Doctoral Fellowship Program No. 2/40 (31)/ 2015/ RD-II/9474.

\section{REFERENCES}

[1] O. L. Mangasarian, 'Second and higher-order duality in nonlinear programming," J. Math. Anal. Appl., vol. 51, 1975, pp. 607-620.

[2] B. Mond and J. Zhang, "Higher-order invexity and duality in mathematical programming," Generalized Convexity, Generalized Monotonicity: Recent Results, Kluwer Academic, Dordrecht, pp. 357-372, 1998.

[3] X. Chen, "Higher-order symmetric duality in nondifferentiable multiobjective programming problems," J. Math. Anal. Appl., vol. 290, 2004, pp. 423-435.

[4] S. K. Mishra, "Second-order symmetric duality in mathematical programming with F -convexity," European J. Oper. Res., vol. 127, 2000, pp. 507-518.

[5] B. Mond, Generalized Concavity in Optimization and Economics Academic Press, New York, 1981, pp. 263-279.

[6] I. Ahmad, Z. Husain, and S. Sharma, "Higher-order duality in non-differentiable multiobjective programming," Numer. Func. Anal. Opt., vol. 28, pp. 989-1002, 2007.

[7] I. Ahmad, "Unified higher-order duality in nondifferentiable multiobjective programming involving cones," Math. Comput. Model, vol. 55, no. 3-4, pp. 419-425, 2012.

[8] Z. Xu, "Mixed type duality in multiobjective programming problems," J. Math. Anal. Appl., vol. 198, 1996, pp. 621-635.

[9] I. Ahmad and Z. Husain, "Multiobjective mixed symmetric duality involving cones Comput," Math. Appl., vol. 59, 2010, pp. 319-326.

[10] S. Chandra, I. Husain, and Abha, "On mixed symmetric duality in mathematical programming," Opsearch, vol. 36, no. 2, pp. 165-171, 1999.

[11] X. M. Yang, K. L. Teo, and X. Q. Yang, "Mixed symmetric duality in nondifferentiable mathematical programming," Indian Journal of Pure and Applied Mathematics, vol. 34, no. 5, pp. 805-815, 2003.

[12] I. Ahmad, "Multiobjective mixed symmetric duality with invexity," New Zealand Journal of Mathematics, vol. 34, no. 1, pp. 1-9, 2005.

[13] C. R. Bector, S. Chandra, and Abha, "On mixed symmetric duality in multiobjective programming," Opsearch, vol. 36, no. 4, pp. 399-407, 1999.

[14] S. Chandra and V. Kumar, "A note on pseudo-invexity and symmetric duality," European J. Oper. Res., vol. 105, 1998, pp. 626-629.

[15] T. R. Gulati, I. Ahmad, and I. Husain, "Second-order symmetric duality with generalized convexity," Opsearch, vol. 38, 2001, pp. 210-222.

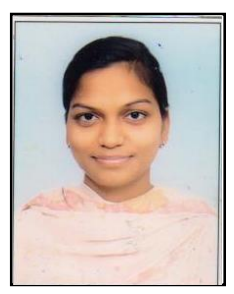

Khushboo Verma is currently pursuing her post doctoral degree in mathematics at University of Lucknow, Lucknow. She received her Ph.D degree from Indian Institute of Technology Roorkee, Roorkee, India and her master of science in mathematics from Banaras Hindu University, Varanasi, India. She has published 11 research papers in journal of international repute.

Pankaj Mathur is a professor in the Department of Mathematics \& Astronomy, University of Lucknow, Lucknow, India. His research interests are in the areas of approximation theory and optimization.

T. R. Gulati is a professor in the Department of Mathematics, Indian Institute of Technology Roorkee, India. His research interests are in the areas of single and multiobjective mathematical programming and generalized convexity. He has published more than 70 research papers in journal of international repute. 